\title{
Histochemical Structure of the Mucus Gel Layer Coating the Fecal Surface of Rodents, Rabbits and Humans
}

\author{
Akira ShImoтoyodome ${ }^{1}$, Shinichi Meguro ${ }^{1}$, Ichiro ToKImitsu ${ }^{1}$ and Takashi SAKATA ${ }^{2}$ \\ ${ }^{1}$ Biological Science Laboratories, Kao Corporation, 2606 Akabane, Ichikai-machi, \\ Haga-gun, Tochigi 321-3497, Japan \\ ${ }^{2}$ Department of Basic Sciences, Ishinomaki Senshu University, 1 Shinmito, \\ Minamisakai, Ishinomaki 986-8580, Japan
}

(Received February 4, 2005)

\begin{abstract}
Summary The aim of this study was to investigate whether a mucus layer covers the surface of various animal feces and, if so, to show the structure and mucin composition of this layer. The freshly excreted feces of mice, rats, guinea pigs, rabbits and humans were fixed with Carnoy solution. Cross-sections approximately $5 \mu \mathrm{m}$ thick were stained with alcian blue (AB) or the periodic acid Schiff (PAS) reaction. We measured the thickness of the mucus layer on the fecal surface of these sections. The fecal surface was covered with a continuous mucus layer in all specimens. The mucus layers of mice, rats and humans consisted of the alternate stratification of AB-positive and PAS-positive mucin layers. In contrast, the mucus layer consisted of an inner PAS-positive neutral mucin layer and an outer AB-positive acidic mucin layer in guinea pigs and rabbits. The average thicknesses of the mucus layers upon the fecal surfaces for mice, rats, guinea pigs, rabbits and humans were $19 \pm 12,22 \pm 14$, $15 \pm 4.6,19 \pm 14$ and $17 \pm 11 \mu \mathrm{m}$, respectively. These results demonstrated that the fecal surfaces are covered with continuous mucus layers in rodents, rabbits and humans, with substructures varying among species.
\end{abstract}

Key Words feces, human, mucus, rodents, rabbits

Mucus is a complex secretion of the gastrointestinal, tracheobronchial and urogenital epithelia (1). This viscous secretion is $95 \%$ water by weight and contains electrolytes, proteins, nucleic acids, enzymes, immunoglobulins, and mucins (2). Mucins are high molecular weight glycoproteins responsible for the gel-forming properties of mucus (3). Its visco-elastic nature enables mucin to lubricate and protect the above epithelia from enzymatic degradation, acids and noxious agents. Mucin also aggregates microorganisms to facilitate their removal and acts as a substrate for intestinal bacteria (1).

Large intestinal mucosa is characterized by abundant mucus producing cells, i.e. goblet and vacuolated cells (4). Mucus secreted from the large-intestinal mucosa is thought to play an important role in the smooth movement of feces in the colon (5). We histochemically demonstrated a mucus gel layer between the fecal pellets and the wall of the distal colon of rats $(4,6)$. The mucus gel layer on the fecal surface may also function as a lubricant for fecal output from the rectum.

However, there have been no reports on the existence of a mucus gel layer on the surface of excreted feces, or on its histochemical structure. Thus, we investigated if such a mucus gel layer covers the surface of feces excreted by rodents, rabbits and humans as well as the structure and mucin composition of this layer.

E-mail: shimotoyodome.akira@ kao.co.jp

\section{Materials and Methods}

Animals. We purchased 6-wk-old male C57BL/6J mice from CLEA Japan, Inc. (Tokyo, Japan), 7-wk-old male Sprague-Dawley rats from Japan SLC, Inc. (Hamamatsu, Japan), male brownish guinea pigs (weighing 400-500 g) from Saitama Experimental Animal Supply (Saitama, Japan), and male New Zealand White (NZW) rabbits (weighing 2.0-2.49 kg) from Oriental Yeast Co. Ltd. (Tokyo, Japan). We housed all animals in individual cages in a room maintained at $23 \pm 2.0^{\circ} \mathrm{C}$, with the relative humidity set at $55 \pm 10 \%$ and a daily photo period of 07.00-19.00 h. Each animal had free access to a standard diet for the maintenance of laboratory animals (CE-2 for mice and rats, CR-3 for rabbits, CLEA Japan, Inc.; Labo RG-RO for guinea pigs, Nosan Corporation, Yokohama, Japan) and to drinking water.

All animal experiments were conducted in the Experimental Animal Facility of Kao Tochigi Institute. The Animal Care Committee of Kao Tochigi Institute approved the present study. All experiments strictly followed the guidelines of that committee. Officially qualified animal care personnel monitored the handling of animals.

Light microscopic observation of feces

Animal feces. We used three animals from each species. We collected a fecal pellet just after its excretion from each animal and placed these pellets in ice-cooled Carnoy solution (absolute ethanol : chloroform : acetic anhydride $=6: 3: 1, \mathrm{v} / \mathrm{v}$ ) for $30 \mathrm{~min}$ and then they were 
A

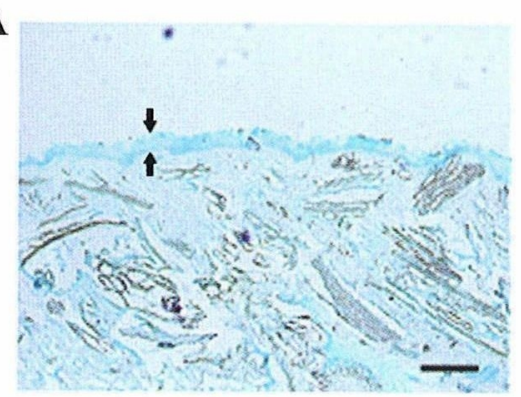

B

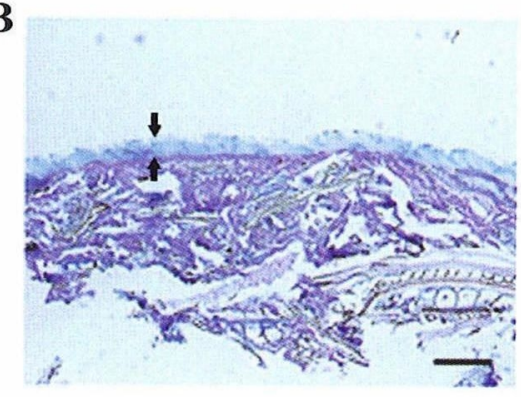

C

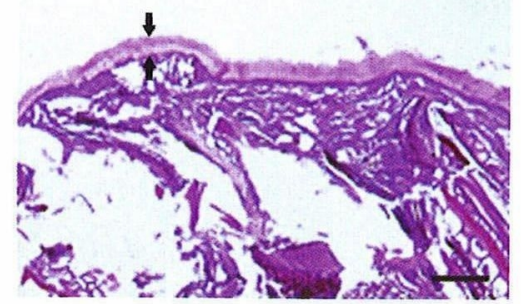

Fig. 1. Light micrograph of a cross section of a mouse fecal sample stained with alcian blue at pH 1.0 (A) or $\mathrm{pH} 2.5$ (B), or with the periodic acid Schiff reaction $(C)$. bar $=50 \mu \mathrm{m}$. $\downarrow$, surface of the mucus gel layer; $\uparrow$, boundary between the mucus gel layer and feces.

A

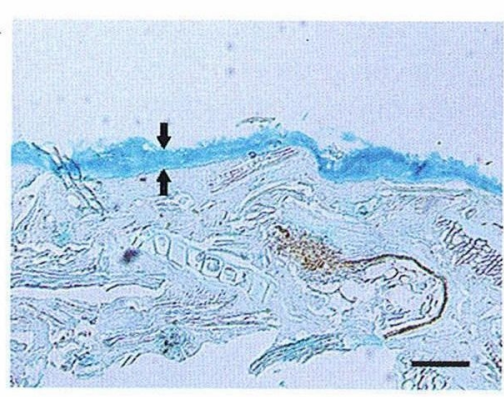

B

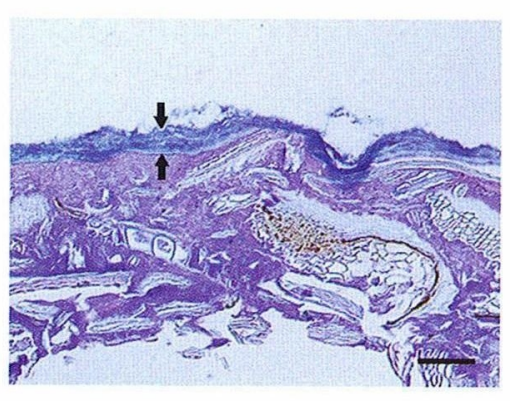

C

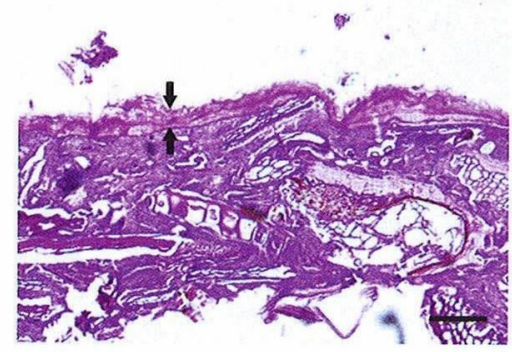

Fig. 2. Light micrograph of a cross section of a rat fecal sample stained with alcian blue at $\mathrm{pH} 1.0$ (A) or $\mathrm{pH} 2.5$ (B), or with the periodic acid Schiff reaction (C). bar $=50 \mu \mathrm{m}$. $\downarrow$, surface of the mucus gel layer; $\uparrow$, boundary between the mucus gel layer and feces.

A

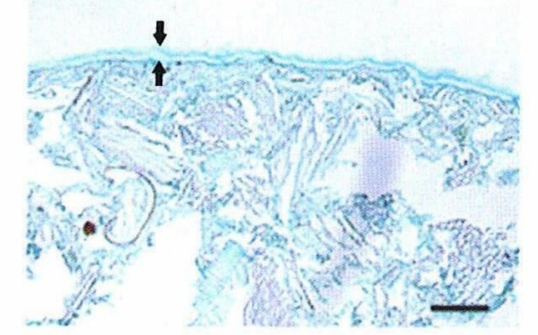

B

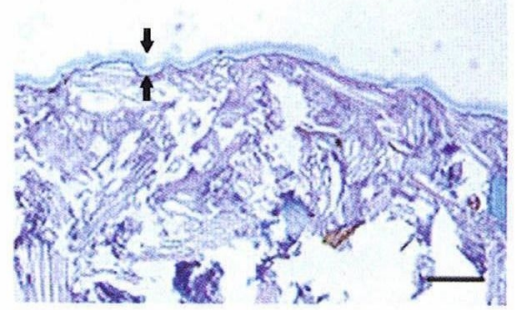

C

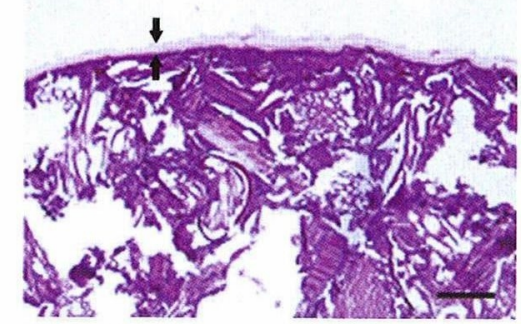

Fig. 3. Light micrograph of a cross section of a guinea pig fecal sample stained with alcian blue at pH 1.0 (A) or $\mathrm{pH} 2.5$ (B), or with the periodic acid Schiff reaction (C). bar $=50 \mu \mathrm{m}$. $\downarrow$, surface of the mucus gel layer; $\uparrow$, boundary between the mucus gel layer and feces.

fixed for $24 \mathrm{~h}$ at $4^{\circ} \mathrm{C}$ in absolute ethanol. Fixed fecal pellets were embedded in paraffin and serially cut into cross sections $5 \mu \mathrm{m}$ thick. The sections were stained with alcian blue $(\mathrm{AB}$, Wako Pure Chemical Industries, Osaka, Japan) at $\mathrm{pH} 1.0$ or 2.5 , or with the periodic acid Schiff (PAS) reaction to detect sulfated glycoprotein (sulfomucin), acidic glycoprotein (sulfomucin and/or sialomucin), or glycoprotein (mucin), respectively (7-9).

We selected six regions per section using a light microscope equipped with a cross-lined eye-piece as follows (6). The approximate center of the cross section of each fecal pellet was fitted to the center of the cross line. The initial angle of the cross section was set arbitrarily. The crossing of the top (12 o'clock) cross line and the lumenal surface of the mucosa was the first site to measure, whereby we measured the thickness of the mucus layer at three measurement points separated by 100 $\mu \mathrm{m}$ intervals in an image captured by a DP-70 CCD camera using DP Controller software (Olympus Corporation, Tokyo, Japan). Then, each specimen was turned clockwise 5 times $\left(60^{\circ}\right.$ per turn) to select five other measurement sites to cover the entire circumference. Thus, we obtained 18 measurements of the thickness of the mucus layer per specimen.

Human feces. A fecal sample was collected from a healthy male volunteer of $30 \mathrm{y}$ old. The protocol and the objective of the study were explained in detail to the subject before the study and written consent produced under free will was obtained. The study protocol was approved by the Ethics Committee of Kao Tochigi Institute.

The subject had neither a history of serious gastrointestinal diseases nor any gastrointestinal symptoms at the time of sampling. The subject had been eat- 
A

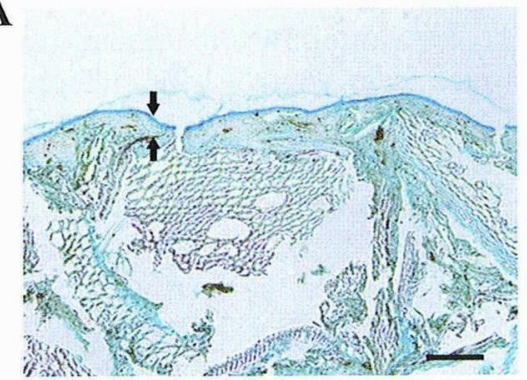

B

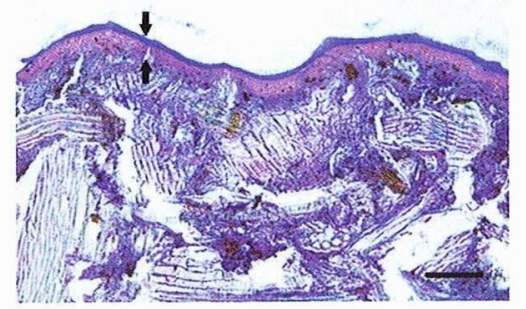

C

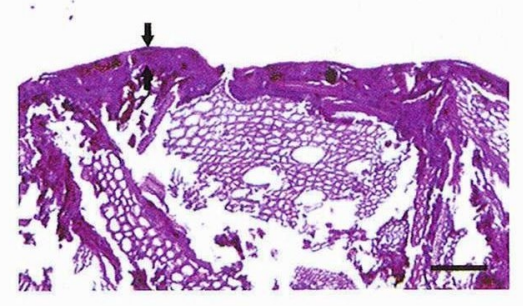

Fig. 4. Light micrograph of a cross section of a rabbit fecal sample stained with alcian blue at pH 1.0 (A) or pH 2.5 (B), or with the periodic acid Schiff reaction (C). bar $=50 \mu \mathrm{m}$. $\downarrow$, surface of the mucus gel layer; $\uparrow$, boundary between the mucus gel layer and feces.

A

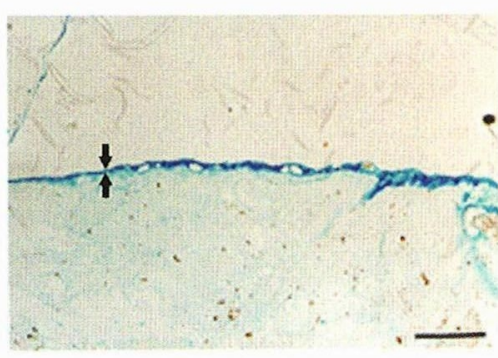

B

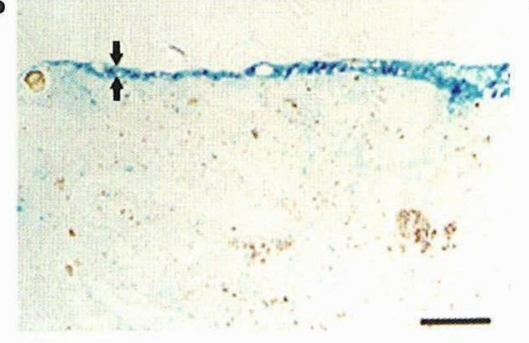

C

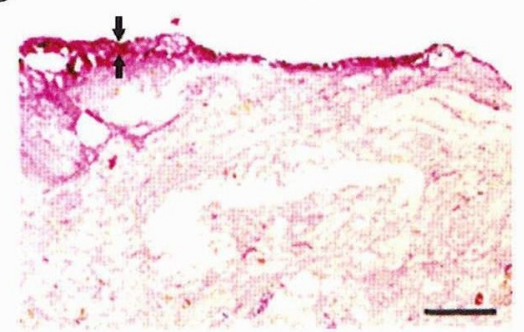

Fig. 5. Light micrograph of a cross section of a human fecal sample stained with alcian blue at pH 1.0 (A) or pH 2.5 (B), or with the periodic acid Schiff reaction (C). bar $=50 \mu \mathrm{m}$. $\downarrow$, surface of the mucus gel layer; $\uparrow$, boundary between the mucus gel layer and feces.

ing an uncontrolled conventional Japanese diet for a few days prior to sampling.

We prepared cylinders approximately $5.4 \mathrm{~mm}$ in diameter and $10 \mathrm{~mm}$ long from gelatin capsules (No. 03, Eli Lilly Japan, Kobe, Japan) by removing both their ends. We carefully inserted three different cylinders vertically into freshly excreted feces placed on a dried paper towel to obtain three samples of approximately 20 $\mathrm{mm}^{2} \times 5 \mathrm{~mm}$. The samples, still inside the gelatin cylinders, were fixed with ice-cooled Carnoy solution for $30 \mathrm{~min}$, and then fixed for $24 \mathrm{~h}$ at $4^{\circ} \mathrm{C}$ in absolute ethanol. The fixed fecal samples in the gelatin capsules were dehydrated with two changes of absolute ethanol and three changes of propylene oxide ( $15 \mathrm{~min}$ each) at room temperature, then embedded in low viscosity Spurr's resin (Polyscience, Inc., Warrington, PA, USA) and polymerized at $70^{\circ} \mathrm{C}$ for $16 \mathrm{~h}$. Cross-sections approximately $5 \mu \mathrm{m}$ thick were cut and stained with $\mathrm{AB}$ at $\mathrm{pH} 1.0$ or 2.5 , or with the PAS reaction as stated above.

We observed each mucus gel layer on the fecal surface using a $\times 10$ objective lens and $\times 10$ eye pieces and measured the thickness of the layer. We selected six measurement sites on three histological sections for each staining. More precisely, we placed each histological specimen with its lumenal side on the top of the observation field. Then, we selected six measurement points from left to right at $500 \mu \mathrm{m}$ intervals using an eye-piece micrometer. We calculated the thickness of the mucus layer at the above measurement points by dividing the stained area by the length of the outer circumference (approximately $500 \mu \mathrm{m}$ ), both of which were measured automatically using an Image Analysis System (Quantimet 500+, Leica Cambridge Ltd., Cambridge, England).

Statistical analysis. Numeric data were expressed as means and their standard deviations. We adopted Scheffe's multiple comparison using the error variance of the preliminary analysis of variance (ANOVA). Differences were considered significant when the error probability was smaller than 0.05 .

\section{Results}

The surfaces of all specimens were covered with continuous mucus layers. The stainability and structure of each layer varied between species.

\section{Mice and Rats}

Continuous mucus gel layers stained with $\mathrm{AB}$ at $\mathrm{pH}$ 1.0 and 2.5, and with the PAS reaction covered the surfaces of the feces of mice (Fig. 1) and rats (Fig. 2). This mucus layer consisted of the stratification of 3 to 20 sub-layers. The inside of the feces was also stained with $\mathrm{AB}$ at $\mathrm{pH} 2.5$ and with the PAS reaction, and was weakly stained with $\mathrm{AB}$ at $\mathrm{pH}$ 1.0. The thicknesses of the mucus gel layers stained with $\mathrm{AB}$ at $\mathrm{pH} 1.0$ or 2.5 , or the PAS reaction were $14 \pm 8,15 \pm 8$, or $19 \pm 12 \mu \mathrm{m}$ (mean $\pm S D$ ), respectively, in mice. The thicknesses of the mucus gel layers stained with $\mathrm{AB}$ at $\mathrm{pH} 1.0$ or 2.5 . or the PAS reaction were $21 \pm 13,22 \pm 13$, or $22 \pm 14 \mu \mathrm{m}$ (mean \pm SD), respectively, in rats. There was no remarkable intra-species variance in mucus gel layer thickness in either species. 


\section{Guinea pigs}

An alcian blue $(\mathrm{pH} 1.0$ and 2.5)-positive outer layer and PAS-positive inner layer covered the surface of the feces of guinea pigs (Fig. 3). Both outer and inner layers were stratified. The inside of the feces was also stained with the PAS reaction, and weakly stained with $\mathrm{AB}$ at pH 1.0 and 2.5. The thicknesses of the mucus gel layers stained with $\mathrm{AB}$ at $\mathrm{pH} 1.0$ or 2.5 , or the PAS reaction were $5.2 \pm 2.0,5.4 \pm 1.8$, or $15 \pm 4.6 \mu \mathrm{m}($ mean $\pm S D)$, respectively. There was no remarkable intra-individual variance in mucus gel layer thickness.

Rabbits

An alcian blue ( $\mathrm{pH} 1.0$ and 2.5) and PAS-positive outer layer and PAS-positive inner layer covered the surface of the feces of rabbits (Fig. 4). Both outer and inner layers had a stratified structure. The inside of the feces was stained with the PAS reaction, and weakly stained with $\mathrm{AB}$ at $\mathrm{pH} 1.0$ and 2.5. The thicknesses of the mucus gel layers stained with $\mathrm{AB}$ at $\mathrm{pH} 1.0$ or 2.5 , or the PAS reaction were $5.5 \pm 2.7,5.8 \pm 2.5$, or $19 \pm 14$ $\mu \mathrm{m}$ (mean $\pm \mathrm{SD}$ ), respectively, without remarkable interindividual variance.

\section{Humans}

A continuous mucus gel layer stained with $\mathrm{AB}$ at $\mathrm{pH}$ 1.0 and 2.5, and with the PAS reaction covered the surface of human feces (Fig. 5). We observed small $(\sim 1$ $\mu \mathrm{m})$ spots stained with $\mathrm{AB}(\mathrm{pH} 1.0$ and 2.5) and many spots stained with the PAS reaction inside the human feces. The thicknesses of the mucus gel layer stained with $\mathrm{AB}$ at $\mathrm{pH} 1.0$ or 2.5 , or the PAS reaction were $17 \pm$ $11,18 \pm 10$, or $18 \pm 11 \mu \mathrm{m}$ (mean $\pm \mathrm{SD})$, respectively.

\section{Discussion}

The present histochemical method used for the human sample effectively preserved the mucus gel layer on the fecal surface.

To our knowledge, this is the first report demonstrating the mucus gel layer covering the fecal surface of various mammalian species, including humans. Although the differences in the thicknesses of the mucus gel layers stained with PAS varied significantly between guinea pigs and rats $(p<0.01)$, the differences among observed species was not large (Figs. 1-5) in spite of the huge differences in fecal size. Dietary control may minimize intra-species variance in mucus gel layer thickness of each experimental animal species.

The above thickness of the fecal mucus layer was not very different from that left on the mucosal surface of the vacant distal colon, or approximately a half of that between the colonic wall and fecal pellets (4). Thus, a relatively thin mucus layer at the fecal surface, together with that attached to the mucosal surface, should be sufficient to lubricate or to function as a diffusion barrier for a wide range of mammals with different body sizes and food habits.

We previously reported that the surface of fecal pellets within the distal colon of rats was covered with stratified layers of sialomucin, and that the outermost layer of the fecal surface coat exclusively consisted of sulfomucin (6). In the present study, such a double-lay- ered structure was not observed at the fecal surface. Instead, stratified layers containing sulfomucin covered the surface of excreted feces (Fig. 2). This sulfomucincontaining mucus gel layer on the excreted feces (21. $13 \mu \mathrm{m}$ in thickness) was as thick as the outer layer of the double-layered fecal surface coat within the distal colon $(20.0 \pm 7.8 \mu \mathrm{m})$ (6). Such a single sulfomucincontaining layer upon the fecal surface was also observed on the excreted feces of mice and humans (Figs. 1 and 5, respectively).

Knowing that there are many mucin-degrading bacteria in the human hindgut (10) and feces (11), it is possible that neutral mucin and sialomucin are already degraded inside the large intestine to leave only sulfomucin to cover the fecal surface when feces are excreted. Alternatively, it is also possible that the mucus gel layer deposited on the fecal surface in the distal colon might be removed during fecal passage and might be replaced by sulfomucin-rich mucus, the most predominant type of mucin secreted in the rectum (12).

Multiple layers of mucin on the surface of excreted feces in mice, rats and humans (Figs. 1, 2 and 5, respectively) suggest the repeated intermittent secretion of mucus after the forming of fecal pellets, either in one place or during movement in the distal colon. The latter should accompany a stop-and-go type movement of the fecal pellet, which is coordinated with mucus secretion.

A double-layered structure consisting of an inner neutral mucin layer and an outer acidic mucin layer was observed in the mucus gel layer of the feces of guinea pigs and rabbits (Figs. 3 and 4), a structure different from that in mice, rats and humans. This difference might come from variation in the physicochemical property of mucus between species. The present results may suggest that the neutral mucin layer, which strongly adhered to the fecal surface, was not degraded and sulfomucin-rich mucus was overlaid during fecal passage in the rectum in guinea pigs and rabbits. However, we do not know if these species are lacking in neutral mucin-degrading bacteria in the colon. It is also unclear if the colonic mucus secretions of these species are intermittent or more-or-less continuous. Furthermore, the AB-positive outer mucus layer was not stained with PAS in guinea pigs (Fig. 3). A high degree of sulfation may explain this attenuated PAS-stainability of the outer acidic mucin layer in guinea pigs.

Matsuo et al. studied the mucous gel layer on the normal mucosa of the human large intestine using Carnoy fixation, and showed a multilaminated structure $(89 \pm 80 \mu \mathrm{m}$ in thickness, mean \pm SE) alternately consisting of sialomucin and sulfomucin in the rectum (13). The mucous gel layer upon the human fecal surface in this study was thinner than the mucosal mucus layer, and contained sulfomucin uniformly (Fig. 5). We do not know if the difference in the mucin composition of this previously reported mucosal mucus layer and the fecal mucus layer reported in the present study is just due to individual variance or to an alteration in the mucin composition during passage. We previously reported that colonic mucus was reduced in rats with 
loperamide-induced constipation (6) and was increased by ingestion of sulfated polysaccharides (14). It remains to be investigated whether colonic or fecal mucus is affected by bowel or dietary conditions in humans.

In the large intestine, the luminal mucin has been suggested to protect colo-rectal mucosa, to lubricate fecal passage and to form a barrier for the transport of solutes $(4,5,12)$. The mucus gel layer on the fecal surface may also function as a lubricant for fecal output from the rectum.

In conclusion, this study histochemically demonstrated that a mucous gel layer covers the entire surface of excreted feces in experimental rodents, rabbits and humans. These results suggest that the mucus gel layer on the fecal surface may function as a lubricant for fecal output from the rectum.

\section{REFERENCES}

1) Neutra MR, Forstner JF. 1987. Gastrointestinal mucins: Synthesis and secretion. In: Physiology of Gastrointestinal Tract, 2nd ed (Johnson LR, ed), p 975-1008. Raven, New York.

2) Satchithanandam S, Klurfeld DM, Calvert RJ, Cassidy MM. 1996. Effects of dietary fibers on gastrointestinal mucin in rats. Nutr Res 16: 1163-1177.

3) Allen A, Hutton A, Pearson JP. 1984. Mucous glycoprotein structure, gel formation and gastrointestinal mucus function. In: Mucus and Mucosa (Sellers LA, ed), p 137151. Pitman, London.

4) Sakata T, Engelhardt WV. 1981. Luminal mucin in the large intestine of mice, rats and guinea pigs. Cell Tissue Res 219: 629-635.

5) Smith B, Butler M. 1974. The autonomic control of colonic mucin secretion in the mouse. Br J Exp Pathol
55: 615-621.

6) Shimotoyodome A, Meguro S, Hase T, Tokimitsu I, Sakata T. 2000. Decreased colonic mucus in loperamide-induced constipation of rat. Comp Biochem Physiol A Mol Integr Physiol 126: 203-212.

7) McManus JFA. 1946. Histological and histochemical use of periodic acid. Stain Technol 23: 99-108.

8) Mowry RW, Winkler CH. 1956. The coloration of acid carbohydrate of bacteria and fungi in the tissue sections with special reference to capsules of Cryptococcus neoformis, Pneumococci, and Staphylococci. Am J Pathol 32: 628-629.

9) Lev R, Spicer SS. 1964. Specific staining of sulfate group with alcian blue at low $\mathrm{pH}$. J Histochem Cytochem 12: 309-310.

10) Corfield AP, Wagner SA, Clamp JR, Kriaris MS, Hoskins LC. 1992. Mucin degradation in the human colon: production of sialidase, sialate $\mathrm{O}$-acetylesterase, $\mathrm{N}$-acetylneuraminate lyase, arylesterase, and glycosulfatase activities by strains of fecal bacteria. Infect Immun $\mathbf{6 0}$ : 3971-3978.

11) Rhodes JM, Black RR, Gallimore R, Savage A. 1985. Histochemical demonstration of desialation and desulphation of normal and inflammatory bowel disease rectal mucus by faecal extracts. Gut 26: 1312-1318.

12) Ueda M. 1983. Histochemical studies on Goblet cells in rat colon during development. J Jpn Soc Colo-Proctology 36: 626-634 (in Japanese).

13) Matsuo K, Ota H, Akamatsu T, Sugiyama A, Katsuyama T. 1997. Histochemistry of the surface mucous gel layer of the human colon. Gut 40: 782-789.

14) Shimotoyodome A, Meguro S, Hase T, Tokimitsu I, Sakata T. 2001. Sulfated polysaccharides, but not cellulose, increase colonic mucus in rats with loperamideinduced constipation. Dig Dis Sci 46: 1482-1489. 